International Mathematical Forum, Vol. 8, 2013, no. 27, 1307 - 1322

HIKARI Ltd, www.m-hikari.com

http://dx.doi.org/10.12988/imf.2013.35108

\title{
Ulam-Hyers Stability of a 2-Variable $A C$-Mixed Type Functional Equation in Felbin's Type Spaces: Fixed Point Method
}

\author{
John M. Rassias ${ }^{1}$, Matina J. Rassias ${ }^{2}$, M. Arunkumar ${ }^{3}$ \\ and T. Namachivayam ${ }^{4}$ \\ ${ }^{1}$ Pedagogical Department E.E. \\ Section of Mathematics and Informatics \\ National and Capodistrian University of Athens \\ 4, Agamemnonos Str., Aghia Paraskevi, Athens 15342, Greece \\ jrassias@primedu.uoa.gr \\ ${ }^{2}$ Department of Statistical Science \\ University College London \\ 1-19 Torrington Place, \#140, London, WC1E 7HB, UK \\ matina@stats.ucl.ac.uk \\ ${ }^{3,4}$ Department of Mathematics, Government Arts College \\ Tiruvannamalai - 606 603, TamilNadu, India \\ annarun2002@yahoo.co.in \\ namachi.siva@rediffmail.com
}

Copyright (C) 2013 John M. Rassias et al. This is an open access article distributed under the Creative Commons Attribution License, which permits unrestricted use, distribution, and reproduction in any medium, provided the original work is properly cited.

Abstract. In this paper, the authors obtain the generalized Ulam - Hyers stability of a 2 variable $\mathrm{AC}$ - mixed type functional equation

$$
f(2 x+y, 2 z+w)-f(2 x-y, 2 z-w)=4[f(x+y, z+w)-f(x-y, z-w)]-6 f(y, w)
$$

in Felbin's type spaces using fixed point method.

Mathematics Subject Classification: 39B52, 32B72, 32B82

Keywords: Additive functional equations, cubic functional equation, Mixed type AC functional equation, Ulam - Hyers stability, Felbin's type spaces, Fixed Point 


\section{INTRODUCTION}

The investigation of stability problems for functional equations is related to the famous Ulam problem [43] (in 1940), concerning the stability of group homomorphisms, which was first solved by D. H. Hyers [13], in 1941. This stability problem was further generalized by several authors $[2,9,28,32,36]$. We cite also other pertinent research works $[1,4,7,11,15,16,18,31,33,37$, $40,46,48]$.

The general solution and Ulam stability of mixed type additive and cubic functional equation of the form

$$
\begin{aligned}
& 3 f(x+y+z)+f(-x+y+z)+f(x-y+z)+f(x+y-z) \\
& \quad+4[f(x)+f(y)+f(z)]=4[f(x+y)+f(x+z)+f(y+z)]
\end{aligned}
$$

introduced by J.M. Rassias [29]. The stability of generalized mixed type functional equation of the form

$$
f(x+k y)+f(x-k y)=k^{2}[f(x+y)+f(x-y)]+2\left(1-k^{2}\right) f(x)
$$

for fixed integers $k$ with $k \neq 0, \pm 1$ in quasi -Banach spaces was investigated by

M. Eshaghi Gordji and H. Khodaie [10]. The mixed type functional equation (1.2) is having the property additive, quadratic and cubic.

The solution and stability of a $n$-dimensional additive functional equation

$$
f\left(a \sum_{i=1}^{n-1} x_{i}-2 a x_{n}\right)+f\left(2 a \sum_{i=1}^{n-1} x_{i}-a x_{n}\right)=3 a\left(\sum_{i=1}^{n-1} f\left(x_{i}\right)-f\left(x_{n}\right)\right) \text { for } n \geq 3
$$

where $a$ are integers, $a \geq 1$ with fixed point Alternative was investigated by K. Ravi, M. Arunkumar [34]. Also, Y.S. Jung, I.S. Chang [17] discussed the Hyers - Ulam - Rassias stability for the cubic functional equation

$$
\begin{aligned}
& f(x+y+2 z)+f(x+y-2 z)+f(2 x)+f(2 y) \\
& \quad=2[f(x+y)+2 f(x+z)+2 f(y+z)+2 f(x-z)+2 f(y-z)]
\end{aligned}
$$

with the fixed point alternative. Infact an $n$-dimensional cubic functional equation

$$
\begin{aligned}
f\left(\sum_{j=1}^{n-1} x_{j}+2 x_{n}\right) & +f\left(\sum_{j=1}^{n-1} x_{j}-2 x_{n}\right)+\sum_{j=1}^{n-1} f\left(2 x_{j}\right) \\
& =2 f\left(\sum_{j=1}^{n-1} 2 x_{j}\right)+4 \sum_{j=1}^{n-1}\left(f\left(x_{j}+x_{n}\right)+f\left(x_{j}-x_{n}\right)\right)
\end{aligned}
$$

and its Hyers - Ulam - Rassias stability with the help of alternative fixed point idea was dealt by H.Y. Chu and D.S. Kang [5]. Recently, F. Moradlou et al., [25] proved the stability of Cauchy functional equation

$$
f(x+y)=f(x)+f(y)
$$

in Felbin's type spaces using fixed point approach.

J.H. Bae and W.G. Park [6] proved the general solution of the 2- variable quadratic functional equation

$$
f(x+y, z+w)+f(x-y, z-w)=2 f(x, z)+2 f(y, w)
$$


and investigated the generalized Hyers-Ulam-Rassias stability of (1.7). The above functional equation has solution

$$
f(x, y)=a x^{2}+b x y+c y^{2} .
$$

Very recently, M. Arunkumar etal., [3] first time introduced and investigated the solution and generalized Ulam-Hyers stability of a 2 - variable AC - mixed type functional equation

$$
f(2 x+y, 2 z+w)-f(2 x-y, 2 z-w)=4[f(x+y, z+w)-f(x-y, z-w)]-6 f(y, w)
$$

having solutions

$$
f(x, y)=a x+b y
$$

and

$$
f(x, y)=a x^{3}+b x^{2} y+c x y^{2}+d y^{3}
$$

in Banach space via direct and fixed point approach.

The solution of the $\mathrm{AC}$ functional equation (1.9) is given in the following lemmas.

Lemma 1.1. [3] If $f: U^{2} \rightarrow V$ be a mapping satisfying (1.9) and let $g: U^{2} \rightarrow V$ be a mapping given by

$$
g(x, x)=f(2 x, 2 x)-8 f(x, x)
$$

for all $x \in U$ then

$$
g(2 x, 2 x)=2 g(x, x)
$$

for all $x \in U$ such that $g$ is additive.

Lemma 1.2. [3] If $f: U^{2} \rightarrow V$ be a mapping satisfying (1.9) and let $h: U^{2} \rightarrow V$ be a mapping given by

$$
h(x, x)=f(2 x, 2 x)-2 f(x, x)
$$

for all $x \in U$ then

$$
h(2 x, 2 x)=8 h(x, x)
$$

for all $x \in U$ such that $h$ is cubic.

Remark 1.3. [3] If $f: U^{2} \rightarrow V$ be a mapping satisfying (1.9) and let $g, h: U^{2} \rightarrow V$ be a mapping defined in (1.12) and (1.14) then

$$
f(x, x)=\frac{1}{6}(h(x, x)-g(x, x))
$$

for all $x \in U$.

In this paper, the authors established the generalized Ulam-Hyers stability using fixed point method in Felbin's type spaces is discussed in Section 3. 


\section{FUZZY REAL NUMBER}

In this section, we give some preliminaries in the theory of fuzzy real numbers. Furthermore, we give some definition which help to investigate the stability in Felbin's type normed linear spaces.

In [12] Grantner takes the fuzzy real number as a decreasing mapping from the real line to the unit interval or lattice in general. Lowen [23] applies the fuzzy real numbers as non-decreasing, left continuous mapping from the real line to the unit interval so that its supremum over $\mathbb{R}$ is 1 . Also fuzzy arithmetic operations on $L$-fuzzy real line were studied by Rodabaugh [39], where he showed that the binary addition is the only extension of addition to $\mathbb{R}((L))$.

Hoehle [14] especially emphasized the role of fuzzy real numbers as modeling a fuzzy threshold softening the notion of Dedekind cut. In this paper a fuzzy real number is taken as a fuzzy normal and convex mapping from the real line to the unit interval. The concept of the fuzzy metric space has been studied by Kaleva [19, 20] by using fuzzy number as a fuzzy set on the real axis. Kaleva also has recently showed that a fuzzy metric space can be embedded in a complete fuzzy metric space [21].

In [8], Felbin introduced the concept of fuzzy normed linear space (FNLS); Xiao and Zhu [44] studied its linear topological structures and some basic properties of a fuzzy normed linear space. It is known that theories of classical normed space and Menger probabilistic normed spaces are special cases of fuzzy normed linear spaces.

Let $\eta$ be a fuzzy subset on $\mathbb{R}$, i.e., a mapping $\eta: \mathbb{R} \rightarrow[0,1]$ associating with each real number $t$ its grade of membership $\eta t$.

Definition 2.1. [8] A fuzzy subset $\eta$ on $R$ is called a fuzzy real number, whose $\alpha$-level set is denoted by $[\eta]_{\alpha}$

$$
\text { i.e., }[\eta]_{\alpha}=\{t: \eta(t) \geq \alpha\} \text {, }
$$

if it satisfies two axioms:

$(N 1)$ There exists $t_{0} \in \mathbb{R}$ such that $\eta\left(t_{0}\right)=1$.

(N2) For each $\alpha \in(0,1],[\eta]_{\alpha}=\left[\eta_{\alpha}^{-}, \eta_{\alpha}^{+}\right]$where $-\infty<\eta_{\alpha}^{-} \leq \eta_{\alpha}^{+}<+\infty$.

The set of all fuzzy real numbers denoted by $F(\mathbb{R})$. If $\eta \in F(\mathbb{R})$ and $\eta(t)=0$ whenever $t<0$, then $\eta$ is called a non-negative fuzzy real number and $F^{*}(\mathbb{R})$ denotes the set of all non-negative fuzzy real numbers.

The number $\overline{0}$ stands for the fuzzy real number as:

$$
\overline{0}= \begin{cases}t, & t=0 \\ 0, & t \neq 0\end{cases}
$$

Clearly, $\overline{0} \in F^{*}(\mathbb{R})$. Also the set of all real numbers can be embedded in $F(\mathbb{R})$ because if $r \in(-\infty, \infty)$, then $\bar{r} \in F(\mathbb{R})$ satisfies $\bar{r}(t)=\overline{0}(t-r)$.

Definition 2.2. [8] Fuzzy arithmetic operations $\oplus, \ominus, \otimes, \oslash$ on $F(\mathbb{R}) \times F(\mathbb{R})$ can be defined as:

(1) $(\eta \oplus \delta)(t)=\sup _{s \in \mathbb{R}}\{\eta(s) \wedge \delta(t-s)\}, t \in \mathbb{R}$

(2) $(\eta \ominus \delta)(t)=\sup _{s \in \mathbb{R}}\{\eta(s) \wedge \delta(s-t)\}, t \in \mathbb{R}$,

(3) $(\eta \otimes \delta)(t)=\sup _{s \in \mathbb{R}}\{\eta(s) \wedge \delta(t / s)\}, t \in \mathbb{R}$

(4) $(\eta \oslash \delta)(t)=\sup _{s \in \mathbb{R}}\{\eta(s t) \wedge \delta(s)\}, t \in \mathbb{R}$. 
The additive and multiplicative identities in $F(\mathbb{R})$ are $\overline{0}$ and $\overline{1}$, respectively. Let $\ominus \eta$ be defined as $\overline{0} \ominus \eta$. It is clear that $\eta \ominus \delta=\eta \oplus(\ominus \delta)$.

Definition 2.3. [8] For $k \in \mathbb{R} 0$, fuzzy scalar multiplication $k \odot \eta$ is defined as $(k \odot \eta)(t)=\eta(t / k)$ and $0 \odot \eta$ is defined to be 0 .

Lemma 2.4. Let $\eta, \delta$ be fuzzy real numbers. Then

$$
\forall t \in \mathbb{R}, \eta(t)=\delta(t) \Leftrightarrow \forall \alpha \in(0,1],[\eta]_{\alpha}=[\delta]_{\alpha} .
$$

Lemma 2.5. Let $\eta, \delta \in F(\mathbb{R})$ and $[\eta]_{\alpha}=\left[\eta_{\alpha}^{-}, \eta_{\alpha}^{+}\right],[\delta]_{\alpha}=\left[\delta_{\alpha}^{-}, \delta_{\alpha}^{+}\right]$. Then

(i) $[\eta \oplus \delta]_{\alpha}=\left[\eta_{\alpha}^{-}+\delta_{\alpha}^{-}, \eta_{\alpha}^{+}+\delta_{\alpha}^{+}\right]$

(ii) $[\eta \ominus \delta]_{\alpha}=\left[\eta_{\alpha}^{-}-\delta_{\alpha}^{-}, \eta_{\alpha}^{+}-\delta_{\alpha}^{+}\right]$,

(iii) $[\eta \otimes \delta]_{\alpha}=\left[\eta_{\alpha}^{-} \delta_{\alpha}^{-}, \eta_{\alpha}^{+} \delta_{\alpha}^{+}\right], \eta, \delta \in F^{*}(\mathbb{R})$,

(iv) $[\overline{1} \oslash \delta]_{\alpha}=\left[1 / \delta_{\alpha}^{+}, 1 / \delta_{\alpha}^{-}\right], \delta_{\alpha}^{-}>0$.

Definition 2.6. [8] Define a partial ordering $\preceq$ in $F(\mathbb{R})$ by $\eta \preceq \delta$ if and only if $\eta_{\alpha}^{-} \leq \delta_{\alpha}^{-}$and $\eta_{\alpha}^{+} \leq \delta_{\alpha}^{+}$for all $\alpha \in(0,1]$. The strict inequality in $F(\mathbb{R})$ is defined by $\eta \prec \delta$ if and only if $\eta_{\alpha}^{-}<\delta_{\alpha}^{-}$and $\eta_{\alpha}^{+}<\delta_{\alpha}^{+}$for all $\alpha \in(0,1]$.

Definition 2.7. [44] Let $X$ be a real linear space, $L$ and $\mathbb{R}$ (respectively, left norm and right norm) be symmetric and non-decreasing mappings from $[0,1] \times[0,1] \rightarrow[0,1]$ satisfying $L(0,0)=$ $0, \mathbb{R}(1,1)=1$. Then $\|\cdot\|$ is called a fuzzy norm and $(X,\|\cdot\|, L, \mathbb{R})$ is a fuzzy normed linear space (abbreviated to FNLS) if the mapping $\|\cdot\|: X \rightarrow F^{*}(R)$ satisfies the following axioms, where $[\|x\|]_{\alpha}=\left[\|x\|_{\alpha}^{-},\|x\|_{\alpha}^{+}\right]$for $x \in X$ and $\alpha \in(0,1]$ :

$(A 1)\|x\|=0$ if and only if $x=0$,

(A2) $\|r x\|=|r| \odot\|x\|$ for all $x \in X$ and $r \in(-\infty, \infty)$,

$(A 3)$ For all $x, y \in X$,

$(A 3 L)$ if $s \leq\|x\|_{1}^{-}, t \leq\|y\|_{1}^{-}$and $s+t \leq\|x \mid+y\|_{1}^{-}$, then $\|x+y\|(s+t) \geq L(\|x\|(s),\|y\|(t))$, $(A 3 R)$ if $s \geq\|x\|_{1}^{-}, t \geq\|y\|_{1}^{-}$and $s+t \geq\|x \mid+y\|_{1}^{-}$, then $\|x+y\|(s+t) \leq L(\|x\|(s),\|y\|(t))$,

Lemma 2.8. [45] Let $(X,\|\cdot\|, L, R)$ be an $F N L S$, and suppose that

(R1) $R(a, b) \leq \max (a, b)$,

$(R 2) \forall \alpha \in(0,1], \exists \beta \in(0, \alpha]$ such that $R(\beta, y) \leq \alpha$ for all $y \in(0, \alpha)$,

(R3) $\lim _{a \rightarrow 0^{+}} R(a, a)=0$.

Then $(R 1) \Rightarrow(R 2) \Rightarrow(R 3)$ but not conversely.

Lemma 2.9. [45] Let $(X,\|\cdot\|, L, R)$ be an FNLS. Then we have the following:

(A) If $R(a, b) \leq \max (a, b)$, then $\forall \alpha \in(0,1],\|x+y\|_{\alpha}^{+} \leq\|x\|_{\alpha}^{+}+\|y\|_{\alpha}^{+}$for all $x, y \in X$.

(B) If $(R 2)$ then for each $\alpha \in(0,1]$ there is $\beta \in(0, \alpha]$ such that $\|x+y\|_{\alpha}^{+} \leq\|x\|_{\beta}^{+}+\|y\|_{\alpha}^{+}$for all $x, y \in X$.

(C) If $\lim _{a \rightarrow 0^{+}} R(a, a)=0$, then for each $\alpha \in(0,1]$ there is $\beta \in(0, \alpha]$ such that $\|x+y\|_{\alpha}^{+} \leq$ $\|x\|_{\beta}^{+}+\|y\|_{\beta}^{+}$for all $x, y \in X$.

Lemma 2.10. [45] Let $(X,\|\cdot\|, L, R)$ be an FNLS, and suppose that

(L1) $L(a, b) \geq \min (a, b)$,

(L2) $\forall \alpha \in(0,1], \exists \beta \in[\alpha, 1]$ such that $L(\beta, \gamma) \geq \alpha$ for all $\gamma \in[\alpha, 1)$,

(L3) $\lim _{a \rightarrow 1^{-}} L(a, a)=1$.

Then $(L 1) \Rightarrow(L 2) \Rightarrow(L 3)$. 
Lemma 2.11. [45] Let $(X,\|\cdot\|, L, R)$ be an FNLS. Then we have the following:

(A) If $L(a, b) \geq \min (a, b)$, then $\forall \alpha \in(0,1],\|x+y\|_{\alpha}^{-} \leq\|x\|_{\alpha}^{-}+\|y\|_{\alpha}^{-}$for all $x, y \in X$.

(B) If (L2) then for each $\alpha \in(0,1]$ there is $\beta \in[\alpha, 1]$ such that $\|x+y\|_{\alpha}^{-} \leq\|x\|_{\beta}^{-}+\|y\|_{\alpha}^{-}$for all $x, y \in X$.

(C) If $\lim _{a \rightarrow 1^{-}} R(a, a)=1$, then for each $\alpha \in(0,1]$ there is $\beta \in[\alpha, 1]$ such that $\|x+y\|_{\alpha}^{-} \leq$ $\|x\|_{\beta}^{-}+\|y\|_{\beta}^{-}$for all $x, y \in X$.

Lemma 2.12. [45] Let $(X,\|\cdot\|, L, R)$ be an FNLS. Then:

(a) If $R(a, b) \geq \max (a, b)$ and $\forall \alpha \in(0,1],\|x+y\|_{\alpha}^{+} \leq\|x\|_{\alpha}^{+}+\|y\|_{\alpha}^{+}$for all $x, y \in X$ then $(A 3 R)$.

(b) If $L(a, b) \leq \min (a, b)$ and $\forall \alpha \in(0,1]\|x+y\|_{\alpha}^{-} \leq\|x\|_{\alpha}^{-}+\|y\|_{\alpha}^{-}$for all $x, y \in X$ then $(A 3 L)$.

Theorem 2.13. [41] Let $(X,\|\cdot\|, L, R)$ be an FNLS and $\lim _{a \rightarrow 0^{+}} R(a, a)=0$. Then $(X,\|\cdot\|, L, R)$ is a Hausdorff topological vector space, whose neighborhood base of origin is $\{N(\epsilon, \alpha): \epsilon>0, \alpha \in$ $(0,1]\}$, where $N(\epsilon, \alpha)=\left\{x:\|x\|_{\alpha}^{+} \leq \epsilon\right\}$.

Definition 2.14. Let $(X,\|\cdot\|, L, R)$ be an FNLS. A sequence $\left\{x_{n}\right\}_{n=1}^{\infty} \subseteq X$ converges to $x \in X$, if $\lim _{n \rightarrow \infty}\left\|x_{n}-x\right\|_{\alpha}^{+}$, for every $\alpha \in(0,1]$ denoted by $\lim _{n \rightarrow \infty} x_{n}=x$.

Definition 2.15. Let $(X,\|\cdot\|, L, R)$ be an FNLS. A sequence $\left\{x_{n}\right\}_{n=1}^{\infty} \subseteq X$ is called a Cauchy sequence if $\lim _{m, n \rightarrow \infty}\left\|x_{m}-x_{n}\right\|_{\alpha}^{+}=0$ for every $\alpha \in(0,1]$.

Definition 2.16. Let $(X,\|\cdot\|, L, R)$ be an FNLS. A subset $A \subseteq X$ is said to be complete if every Cauchy sequence in $A$, converges in $A$. The fuzzy normed space $(X,\|\cdot\|, L, R)$ is said to be a fuzzy Banach space if it is complete.

\section{STABILITY RESULTS: FIXED POINT METHOD}

In this section, we apply a fixed point method for achieving stability of the 2-variable AC functional equation (1.9).

Now, we present the following theorem due to B. Margolis and J.B. Diaz [24] for fixed point Theory.

Theorem 3.1. [24] Suppose that for a complete generalized metric space $(\Omega, \delta)$ and a strictly contractive mapping $T: \Omega \rightarrow \Omega$ with Lipschitz constant $L$. Then, for each given $x \in \Omega$, either

$$
d\left(T^{n} x, T^{n+1} x\right)=\infty \forall n \geq 0,
$$

or there exists a natural number $n_{0}$ such that

(A1) $d\left(T^{n} x, T^{n+1} x\right)<\infty$ for all $n \geq n_{0}$;

(A2) The sequence $\left(T^{n} x\right)$ is convergent to a fixed to a fixed point $y^{*}$ of $T$

(A3) $y^{*}$ is the unique fixed point of $T$ in the set $\Delta=\left\{y \in \Omega: d\left(T^{n_{0}} x, y\right)<\infty\right\}$;

(A4) $d\left(y^{*}, y\right) \preceq \frac{1}{1-L} d(y, T y)$ for all $y \in \Delta$.

Using the above theorem, we now obtain the generalized Ulam - Hyers stability of (1.9).

Through out this section let $U$ be a normed space and $V$ be a Banach space. Define a mapping $F: U^{2} \rightarrow V$ by

$$
\begin{gathered}
F(x, y, z, w)=f(2 x+y, 2 z+w)-f(2 x-y, 2 z-w)-4 f(x+y, z+w) \\
+4 f(x-y, z-w)]+6 f(y, w)
\end{gathered}
$$

for all $x, y, z, w \in U$. 
Theorem 3.2. Let $F: U^{2} \rightarrow V$ be a mapping for which there exist a function $\varphi: U^{4} \rightarrow F^{*}(R)$ with the condition

$$
\lim _{n \rightarrow \infty} \frac{1}{\mu_{i}^{n}} \varphi\left(\mu_{i}^{n} x, \mu_{i}^{n} y, \mu_{i}^{n} z, \mu_{i}^{n} w\right)_{\alpha}^{+}=0
$$

where $\mu_{i}=2$ if $i=0$ and $\mu_{1}=\frac{1}{2}$ if $i=1$ such that the functional inequality

$$
\|F(x, y, z, w)\|_{\alpha}^{+} \preceq \varphi(x, y, z, w)_{\alpha}^{+}
$$

for all $x, y, z, w \in U$ and $\alpha \in(0,1]$. If there exists $L=L(i)<1$ such that the function

$$
x \rightarrow \gamma(x)_{\alpha}^{+}=\frac{1}{2} \odot \psi(x)_{\alpha}^{+},
$$

has the property

$$
\gamma(x)_{\alpha}^{+} \preceq L \mu_{i} \odot \gamma\left(\mu_{i} x\right)_{\alpha}^{+} .
$$

Then there exists a unique 2-variable additive mapping $A: U^{2} \rightarrow V$ satisfying the functional equation (1.9) and

$$
\|f(2 x, 2 x)-8 f(x, x)-A(x, x)\|_{\alpha}^{+} \preceq \frac{L^{1-i}}{1-L} \gamma(x)_{\alpha}^{+}
$$

for all $x \in U$, where $\psi(x)_{\alpha}^{+}$is defined in (3.9), for all $x \in U$.

Proof. Consider the set

$$
\Omega=\left\{p / p: U^{2} \rightarrow V, p(0,0)=0\right\}
$$

and introduce the generalized metric on $\Omega$,

$$
d(p, q)=d_{\gamma}(p, q)=\inf \left\{K \in(0, \infty):\|p(x, x)-q(x, x)\|_{\alpha}^{+} \preceq K \gamma(x)_{\alpha}^{+}, x \in U, \forall \alpha \in(0,1]\right\} .
$$

It is easy to see that $(\Omega, d)$ is complete.

Define $T: \Omega^{2} \rightarrow \Omega$ by

$$
T p(x, x)=\frac{1}{\mu_{i}} p\left(\mu_{i} x, \mu_{i} x\right)
$$

for all $x \in U$. Now $p, q \in \Omega$,

$$
\begin{aligned}
d(p, q) \preceq K & \Rightarrow\|p(x, x)-q(x, x)\|_{\alpha}^{+} \preceq K \gamma(x)_{\alpha}^{+}, x \in U . \\
& \Rightarrow\left\|\frac{1}{\mu_{i}} p\left(\mu_{i} x, \mu_{i} x\right)-\frac{1}{\mu_{i}} q\left(\mu_{i} x, \mu_{i} x\right)\right\|_{\alpha}^{+} \preceq \frac{1}{\mu_{i}} K \gamma\left(\mu_{i} x\right)_{\alpha}^{+}, x \in U, \\
& \Rightarrow\left\|\frac{1}{\mu_{i}} p\left(\mu_{i} x, \mu_{i} x\right)-\frac{1}{\mu_{i}} q\left(\mu_{i} x, \mu_{i} x\right)\right\|_{\alpha}^{+} \preceq L K \gamma(x)_{\alpha}^{+}, x \in U, \\
& \Rightarrow\|T p(x, x)-T q(x, x)\|_{\alpha}^{+} \preceq L K \gamma(x)_{\alpha}^{+}, x \in U, \\
& \Rightarrow d_{\gamma}(p, q) \preceq L K .
\end{aligned}
$$

This implies $d(T p, T q) \preceq L d(p, q)$, for all $p, q \in \Omega$. i.e., $T$ is a strictly contractive mapping on $\Omega$ with Lipschitz constant $L$.

Letting $(x, y, z, w)$ by $(x, x, x, x)$ in $(3.2)$, we obtain

$$
\|f(3 x, 3 x)-4 f(2 x, 2 x)+5 f(x, x)\|_{\alpha}^{+} \preceq \varphi(x, x, x, x)_{\alpha}^{+}
$$

for all $x \in U$. Replacing $(x, y, z, w)$ by $(x, 2 x, x, 2 x)$ in $(3.2)$, we get

$$
\|f(4 x, 4 x)-4 f(3 x, 3 x)+6 f(2 x, 2 x)-4 f(x, x)\|_{\alpha}^{+} \preceq \varphi(x, 2 x, x, 2 x)_{\alpha}^{+}
$$


for all $x \in U$. Now, from (3.5) and (3.6), we have

$$
\begin{aligned}
\|f(4 x, 4 x)-10 f(2 x, 2 x)+16 f(x, x)\|_{\alpha}^{+} \preceq 4 \odot\|f(3 x, 3 x)-4 f(2 x, 2 x)+5 f(x, x)\|_{\alpha}^{+} \\
+\|f(4 x, 4 x)-4 f(3 x, 3 x)+6 f(2 x, 2 x)-4 f(x, x)\|_{\alpha}^{+} \\
\preceq 4 \odot \varphi(x, x, x, x)_{\alpha}^{+}+\varphi(x, 2 x, x, 2 x)_{\alpha}^{+}
\end{aligned}
$$

for all $x \in U$. From (3.7), we arrive

$$
\|f(4 x, 4 x)-10 f(2 x, 2 x)+16 f(x, x)\|_{\alpha}^{+} \preceq \psi(x)_{\alpha}^{+}
$$

where

$$
\psi(x)_{\alpha}^{+}=4 \odot \varphi(x, x, x, x)_{\alpha}^{+}+\varphi(x, 2 x, x, 2 x)_{\alpha}^{+}
$$

for all $x \in U$. It is easy to see from (3.8) that

$$
\|f(4 x, 4 x)-8 f(2 x, 2 x)-2(f(2 x, 2 x)-8 f(x, x))\|_{\alpha}^{+} \preceq \psi(x)_{\alpha}^{+}
$$

for all $x \in U$. Using (1.12) in (3.10), we obtain

$$
\|g(2 x, 2 x)-2 g(x, x)\|_{\alpha}^{+} \preceq \psi(x)_{\alpha}^{+}
$$

for all $x \in U$. From (3.11), we arrive

$$
\left\|\frac{g(2 x, 2 x)}{2}-g(x, x)\right\|_{\alpha}^{+} \preceq \frac{1}{2} \odot \psi(x)_{\alpha}^{+}
$$

for all $x \in U$. Using (3.3) for the case $i=0$ it reduces to

$$
\left\|\frac{g(2 x, 2 x)}{2}-g(x, x)\right\|_{\alpha}^{+} \preceq L \gamma(x)_{\alpha}^{+}
$$

for all $x \in U$,

$$
\text { i.e., } \quad d_{\psi}(g, T g) \preceq L \Rightarrow d(g, T g) \preceq L \preceq L^{1}<\infty .
$$

Again replacing $x=\frac{x}{2}$ in (3.12), we get,

$$
\left\|g(x, x)-2 g\left(\frac{x}{2}, \frac{x}{2}\right)\right\|_{\alpha}^{+} \preceq \psi\left(\frac{x}{2}\right)_{\alpha}^{+}
$$

Using (3.3) for the case $i=1$ it reduces to

$$
\left\|g(x, x)-2 g\left(\frac{x}{2}, \frac{x}{2}\right)\right\|_{\alpha}^{+} \preceq \gamma(x)_{\alpha}^{+}
$$

for all $x \in U$,

$$
\text { i.e., } \quad d_{\psi}(g, T g) \preceq 1 \Rightarrow d(g, T g) \preceq 1 \preceq L^{0}<\infty .
$$

In both cases, we arrive

$$
d(g, T g) \preceq L^{1-i}
$$

Therefore $(A 1)$ holds.

By $(A 2)$, it follows that there exists a fixed point $A$ of $T$ in $\Omega$ such that

$$
A(x, x)=\lim _{n \rightarrow \infty} \frac{1}{\mu_{i}^{n}}\left(f\left(\mu_{i}^{(n+1)} x, \mu_{i}^{(n+1)} x\right)-8 f\left(\mu_{i}^{n} x, \mu_{i}^{n} x\right)\right)
$$

for all $x \in U$. 
To prove $A: U^{2} \rightarrow V$ is additive. Replacing $(x, y, z, w)$ by $\left(\mu_{i}^{n} x, \mu_{i}^{n} y, \mu_{i}^{n} z, \mu_{i}^{n}\right)$ in $(3.2)$ and dividing by $\mu_{i}^{n}$, it follows from (3.1) that

$$
\begin{aligned}
\|A(x, y, z, w)\|_{\alpha}^{+} & =\lim _{n \rightarrow \infty} \frac{\left\|F\left(\mu_{i}^{n} x, \mu_{i}^{n} y, \mu_{i}^{n} z, \mu_{i}^{n}\right)\right\|_{\alpha}^{+}}{\mu_{i}^{n}} \\
& \preceq \lim _{n \rightarrow \infty} \frac{\varphi\left(\mu_{i}^{n} x, \mu_{i}^{n} y, \mu_{i}^{n} z, \mu_{i}^{n} w\right)_{\alpha}^{+}}{\mu_{i}^{n}}=0
\end{aligned}
$$

for all $x, y, z, w \in U$ i.e., $A$ satisfies the functional equation (1.9).

By $(A 3), A$ is the unique fixed point of $T$ in the set $\Delta=\{A \in \Omega: d(f, A)<\infty\}, A$ is the unique function such that

$$
\|f(2 x, 2 x)-8 f(x, x)-A(x, x)\|_{\alpha}^{+} \preceq K \gamma(x)_{\alpha}^{+}
$$

for all $x \in U$ and $K>0$. Finally by $(A 4)$, we obtain

$$
d(f, A) \preceq \frac{1}{1-L} d(f, T f)
$$

this implies

$$
d(f, A) \preceq \frac{L^{1-i}}{1-L}
$$

which yields

$$
\|f(2 x, 2 x)-8 f(x, x)-A(x, x)\|_{\alpha}^{+} \preceq \frac{L^{1-i}}{1-L} \gamma(x)_{\alpha}^{+}
$$

this completes the proof of the theorem.

The following Corollary is an immediate consequence of Theorem 3.2 concerning the stability of (1.9).

Corollary 3.3. Let $F: U^{2} \rightarrow V$ be a mapping and there exits real numbers $\lambda$ and $s$ such that

$$
\| F(x, y, z, w)) \|_{\alpha}^{+} \preceq \begin{cases}\lambda \otimes\left\{\|x\|^{s} \oplus\|y\|^{s} \oplus\|z\|^{s} \oplus\|w\|^{s}\right\}, & s<1 \text { or } s>1 ; \\ \lambda \otimes\|x\|^{s} \otimes\|y\|^{s} \otimes\|z\|^{s} \otimes\|w\|^{s}, & s<\frac{1}{4} \text { or } s>\frac{1}{4} \\ \lambda \otimes\left\{\|x\|^{s} \otimes\|y\|^{s} \otimes\|z\|^{s} \otimes\|w\|^{s}\right. & \\ \left.\oplus\left\{\|x\|^{4 s} \oplus\|y\|^{4 s} \oplus\|w\|^{4 s} \oplus\|z\|^{4 s}\right\}\right\}, & s<\frac{1}{4} \text { or } s>\frac{1}{4}\end{cases}
$$

for all $x, y, z, w \in U$, then there exists a unique 2- variable additive function $A: U^{2} \rightarrow V$ such that

$$
\|f(2 x, 2 x)-8 f(x, x)-A(x, x)\|_{\alpha}^{+} \preceq\left\{\begin{array}{l}
\lambda_{\alpha}^{+} \otimes \frac{2^{(s-1)}\left(18 \oplus 2^{s+1}\right)\left(\|x\|^{s}\right)_{\alpha}^{+}}{\left|2-2^{s}\right|}, \\
\lambda_{\alpha}^{+} \otimes \frac{2^{(4 s-1)}\left(4 \oplus 2^{2 s}\right)\left(\|x\|^{4 s}\right)_{\alpha}^{+}}{\left|2-2^{4 s}\right|} \\
\lambda_{\alpha}^{+} \otimes \frac{2^{(4 s-1)}\left(22 \oplus 2^{2 s}+2 \odot 2^{4 s}\right)\left(\|x\|^{4 s}\right)_{\alpha}^{+}}{2-2^{4 s}}
\end{array}\right.
$$

for all $x \in U$. 
Proof. Setting

$$
\varphi(x, y, z, w)_{\alpha}^{+}=\left\{\begin{array}{l}
\lambda \otimes\left\{\|x\|^{s} \oplus\|y\|^{s} \oplus\|z\|^{s} \oplus\|w\|^{s}\right\} \\
\lambda \otimes\|x\|^{s} \otimes\|y\|^{s} \otimes\|z\|^{s} \otimes\|w\|^{s} \\
\lambda \otimes\left\{\|x\|^{s} \otimes\|y\|^{s} \otimes\|z\|^{s} \otimes\|w\|^{s} \oplus\left\{\|x\|^{4 s} \oplus\|y\|^{4 s} \oplus\|w\|^{4 s} \oplus\|z\|^{4 s}\right\}\right\}
\end{array}\right.
$$

for all $x, y, z, w \in U$. Now,

$$
\begin{aligned}
& \frac{\varphi\left(\mu_{i}^{n} x, \mu_{i}^{n} y, \mu_{i}^{n} z, \mu_{i}^{n} w\right)}{\mu_{i}^{n}} \\
& =\left\{\begin{array}{l}
\frac{\lambda}{\mu_{i}^{n}} \otimes\left\{\left\|\mu_{i}^{n} x\right\|^{s} \oplus\left\|\mu_{i}^{n} y\right\|^{s} \oplus\left\|\mu_{i}^{n} z\right\|^{s} \oplus\left\|\mu_{i}^{n} w\right\|^{s}\right\}, \\
\frac{\lambda}{\mu_{i}^{n}} \otimes\left\|\mu_{i}^{n} x\right\|^{s} \otimes\left\|\mu_{i}^{n} y\right\|^{s} \otimes\left\|\mu_{i}^{n} z\right\|^{s} \otimes\left\|\mu_{i}^{n} w\right\|^{s} \\
\frac{\lambda}{\mu_{i}^{n}} \otimes\left\{\left\|\mu_{i}^{n} x\right\|^{s} \otimes\left\|\mu_{i}^{n} y\right\|^{s} \otimes\left\|\mu_{i}^{n} z\right\|^{s} \otimes\left\|\mu_{i}^{n} w\right\|^{s}\left\{\left\|\mu_{i}^{n} x\right\|^{4 s} \oplus\left\|\mu_{i}^{n} y\right\|^{4 s} \oplus\left\|\mu_{i}^{n} z\right\|^{4 s} \oplus\left\|\mu_{i}^{n} w\right\|^{4 s}\right\}\right\}
\end{array}\right. \\
& =\left\{\begin{array}{l}
\rightarrow 0 \text { as } n \rightarrow \infty, \\
\rightarrow 0 \text { as } n \rightarrow \infty, \\
\rightarrow 0 \text { as } n \rightarrow \infty .
\end{array}\right.
\end{aligned}
$$

Thus, (3.1) is holds.

But we have $\gamma(x)_{\alpha}^{+}=\frac{1}{2} \psi(x)_{\alpha}^{+}$has the property $\gamma(x)_{\alpha}^{+} \preceq L \cdot \mu_{i} \odot \gamma\left(\mu_{i} x\right)_{\alpha}^{+}$for all $x \in U$. Hence

$$
\begin{aligned}
\gamma(x)_{\alpha}^{+}=\frac{1}{2} \odot \psi(x)_{\alpha}^{+} & =\frac{1}{2} \odot\left(4 \varphi(x, x, x, x)_{\alpha}^{+}+\varphi(x, 2 x, x, 2 x)_{\alpha}^{+}\right) \\
& =\left\{\begin{array}{l}
\frac{\lambda}{2} \odot\left(18\|x\|^{s} \oplus 2\|2 x\|^{s}\right), \\
\frac{\lambda}{2} \odot\left(4 \oplus 2^{2 s}\right)\|x\|^{4 s}, \\
\frac{\lambda}{2} \odot\left(22 \oplus 2^{2 s}+2 \odot 2^{4 s}\right)\|x\|^{4 s} .
\end{array}\right.
\end{aligned}
$$

Now,

$$
\begin{aligned}
\frac{1}{\mu_{i}} \gamma\left(\mu_{i} x\right)_{\alpha}^{+}=\left\{\begin{array}{l}
\frac{\lambda}{2 \mu_{i}} \odot\left\{\left(18\left\|\mu_{i} x\right\|^{s} \oplus 2\left\|2 \mu_{i} x\right\|^{s}\right)\right\}, \\
\frac{\lambda}{2 \mu_{i}} \odot\left\{\left(4 \oplus 2^{2 s}\right)\right\}\left\|\mu_{i} x\right\|^{4 s}, \\
\frac{\lambda}{2 \mu_{i}} \odot\left\{\left(22 \oplus 2^{2 s} \oplus 2 \odot 2^{4 s}\right)\right\}\left\|\mu_{i} x\right\|^{4 s} .
\end{array}\right. & =\left\{\begin{array}{l}
\mu_{i}^{s-1} \frac{\lambda}{2} \odot\left(18 \oplus 2^{s+1}\right)\|x\|^{s}, \\
\mu_{i}^{4 s-1} \frac{\lambda}{2} \odot\left(4 \oplus 2^{2 s}\right)\|x\|^{4 s}, \\
\mu_{i}^{4 s-1} \frac{\lambda}{2} \odot\left(22 \oplus 2^{2 s} \oplus 2 \odot 2^{4 s}\right)\|x\|^{4 s} .
\end{array}\right. \\
& =\left\{\begin{array}{l}
\mu_{i}^{s-1} \psi(x)_{\alpha}^{+}, \\
\mu_{i}^{4 s-1} \psi(x)_{\alpha}^{+}, \\
\mu_{i}^{4 s-1} \psi(x)_{\alpha}^{+} .
\end{array}\right.
\end{aligned}
$$

Hence the inequality (3.3) holds either, $L=2^{s-1}$ for $s<1$ if $i=0$ and $L=\frac{1}{2^{s-1}}$ for $s>1$ if $i=1$. Now from (3.4), we prove the following cases for condition $(i)$. 
Case: $1 L=2^{s-1}$ for $s<1$ if $i=0$

$$
\begin{aligned}
\|f(2 x, 2 x)-8 f(x, x)-A(x, x)\|_{\alpha}^{+} & \preceq \lambda_{\alpha}^{+} \otimes \frac{\left(2^{(s-1)}\right)^{1-0}}{1-2^{(s-1)}}\left\{\frac{18 \oplus 2^{(s+1)}}{2}\right\}\left(\|x\|^{s}\right)_{\alpha}^{+} \\
& =\lambda_{\alpha}^{+} \otimes \frac{2^{(s-1)}}{1-2^{(s-1)}}\left\{\frac{18 \oplus 2^{(s+1)}}{2}\right\}\left(\|x\|^{s}\right)_{\alpha}^{+} \\
& =\lambda_{\alpha}^{+} \otimes \frac{2^{(s-1)} \cdot 2}{2-2^{s}}\left\{\frac{18 \oplus 2^{(s+1)}}{2}\right\}\left(\|x\|^{s}\right)_{\alpha}^{+} \\
& =\lambda_{\alpha}^{+} \otimes \frac{2^{(s-1)}\left(18 \oplus 2^{(s+1)}\right)\left(\|x\|^{s}\right)_{\alpha}^{+}}{2-2^{s}}
\end{aligned}
$$

Case:2 $L=\frac{1}{2^{s-1}}$ for $s>1$ if $i=1$

$$
\begin{aligned}
\|f(2 x, 2 x)-8 f(x, x)-A(x, x)\|_{\alpha}^{+} & \preceq \lambda_{\alpha}^{+} \otimes \frac{\left(\frac{1}{2^{(s-1)}}\right)^{1-1}}{1-\frac{1}{2^{(s-1)}}}\left\{\frac{18 \oplus 2^{(s+1)}}{2}\right\}\left(\|x\|^{s}\right)_{\alpha}^{+} \\
& =\lambda_{\alpha}^{+} \otimes \frac{2^{(s-1)}}{2^{(s-1)}-1}\left\{\frac{18 \oplus 2^{(s+1)}}{2}\right\}\left(\|x\|^{s}\right)_{\alpha}^{+} \\
& =\lambda_{\alpha}^{+} \otimes \frac{2^{(s-1)} \cdot 2}{2^{s}-2}\left\{\frac{18 \oplus 2^{(s+1)}}{2}\right\}\left(\|x\|^{s}\right)_{\alpha}^{+} \\
& =\lambda_{\alpha}^{+} \otimes \frac{2^{(s-1)}\left(18 \oplus 2^{(s+1)}\right)\left(\|x\|^{s}\right)_{\alpha}^{+}}{2^{s}-2}
\end{aligned}
$$

In similar manner we can prove the following cases $L=2^{4 s-1}$ for $s<\frac{1}{4}$ if $i=0$ and $L=\frac{1}{2^{4 s-1}}$ for $s>\frac{1}{4}$ if $i=1$ for conditions $(i i)$ and (iii) respectively. Hence the proof is complete.

Theorem 3.4. Let $F: U^{2} \rightarrow V$ be a mapping for which there exist a function $\varphi: U^{4} \rightarrow F^{*}(R)$ with the condition

$$
\lim _{n \rightarrow \infty} \frac{1}{\mu_{i}^{3 n}} \varphi\left(\mu_{i}^{n} x, \mu_{i}^{n} y, \mu_{i}^{n} z, \mu_{i}^{n} w\right)_{\alpha}^{+}=0
$$

where $\mu_{i}=2$ if $i=0$ and $\mu_{1}=\frac{1}{2}$ if $i=1$ such that the functional inequality

$$
\|F(x, y, z, w)\|_{\alpha}^{+} \preceq \varphi(x, y, z, w)_{\alpha}^{+}
$$

for all $x, y, z, w \in U$. If there exists $L=L(i)<1$ such that the function

has the property

$$
x \rightarrow \gamma(x)_{\alpha}^{+}=\frac{1}{2} \odot \psi(x)_{\alpha}^{+},
$$

$$
\gamma(x)_{\alpha}^{+} \preceq L \mu_{i}^{3} \odot \gamma\left(\mu_{i} x\right)_{\alpha}^{+} .
$$

Then there exists a unique 2-variable cubic mapping $C: U^{2} \rightarrow V$ satisfying the functional equation (1.9) and

$$
\|f(2 x, 2 x)-2 f(x, x)-C(x, x)\|_{\alpha}^{+} \preceq \frac{L^{1-i}}{1-L} \gamma(x)_{\alpha}^{+}
$$

for all $x \in U$. The mapping $\psi(x)_{\alpha}^{+}$is defined in (3.9) for all $x \in U$. 
Proof. Consider the set

$$
\Omega=\left\{p / p: U^{2} \rightarrow V, p(0,0)=0\right\}
$$

and introduce the generalized metric on $\Omega$,

$$
d(p, q)=d_{\gamma}(p, q)=\inf \left\{K \in(0, \infty):\|p(x, x)-q(x, x)\|_{\alpha}^{+} \preceq K \gamma(x)_{\alpha}^{+}, x \in U\right\} .
$$

It is easy to see that $(\Omega, d)$ is complete. Define $T: \Omega^{2} \rightarrow \Omega$ by

$$
T p(x, x)=\frac{1}{\mu_{i}^{3}} p\left(\mu_{i} x, \mu_{i} x\right),
$$

for all $x \in U$. Now $p, q \in \Omega$,

$$
\begin{aligned}
d(p, q) \preceq K & \Rightarrow\|p(x, x)-q(x, x)\|_{\alpha}^{+} \preceq K \gamma(x)_{\alpha}^{+}, x \in U . \\
& \Rightarrow\left\|\frac{1}{\mu_{i}^{3}} p\left(\mu_{i} x, \mu_{i} x\right)-\frac{1}{\mu_{i}^{3}} q\left(\mu_{i} x, \mu_{i} x\right)\right\|_{\alpha}^{+} \preceq \frac{1}{\mu_{i}^{3}} K \gamma\left(\mu_{i} x\right), x \in U, \\
& \Rightarrow\left\|\frac{1}{\mu_{i}^{3}} p\left(\mu_{i} x, \mu_{i} x\right)-\frac{1}{\mu_{i}^{3}} q\left(\mu_{i} x, \mu_{i} x\right)\right\|_{\alpha}^{+} \preceq L K \gamma(x)_{\alpha}^{+}, x \in U, \\
& \Rightarrow\|T p(x, x)-T q(x, x)\|_{\alpha}^{+} \preceq L K \gamma(x)_{\alpha}^{+}, x \in U, \\
& \Rightarrow d_{\gamma}(p, q) \preceq L K .
\end{aligned}
$$

This implies $d(T p, T q) \preceq L d(p, q)$, for all $p, q \in \Omega$. i.e., $T$ is a strictly contractive mapping on $\Omega$ with Lipschitz constant $L$.

It followes form (3.8) that

$$
\|f(4 x, 4 x)-2 f(2 x, 2 x)-8(f(2 x, 2 x)-2 f(x, x))\|_{\alpha}^{+} \preceq \psi(x)_{\alpha}^{+}
$$

for all $x \in U$. Using (1.14) in (3.21), we obtain

$$
\left\|\frac{h(2 x, 2 x)}{8}-h(x, x)\right\|_{\alpha}^{+} \preceq \frac{\psi(x)_{\alpha}^{+}}{8}
$$

for all $x \in U$. Using (3.22) for the case $i=0$ it reduces to

$$
\left\|\frac{h(2 x, 2 x)}{8}-h(x, x)\right\|_{\alpha}^{+} \preceq L \gamma(x)_{\alpha}^{+}
$$

for all $x \in U$,

$$
\text { i.e., } \quad d_{\psi}(h, T h) \preceq L \Rightarrow d(h, T g) \preceq L \preceq L^{1}<\infty .
$$

Again replacing $x=\frac{x}{2}$ in (3.22), we get,

$$
\left\|h(x, x)-8 h\left(\frac{x}{2}, \frac{x}{2}\right)\right\|_{\alpha}^{+} \preceq \psi\left(\frac{x}{2}\right)_{\alpha}^{+}
$$

Using (3.23) for the case $i=1$ it reduces to

$$
\left\|h(x, x)-8 h\left(\frac{x}{2}, \frac{x}{2}\right)\right\|_{\alpha}^{+} \preceq \gamma(x)_{\alpha}^{+}
$$

for all $x \in U$,

$$
\text { i.e., } \quad d_{\psi}(h, T h) \preceq 1 \Rightarrow d(h, T h) \preceq 1 \preceq L^{0}<\infty .
$$

In both cases, we arrive

$$
d(h, T h) \preceq L^{1-i} .
$$

Therefore $(A 1)$ holds. The rest of the proof is similar to that of Theorem 3.2. 
The following Corollary is an immediate consequence of Theorem 3.4 concerning the stability of (1.9).

Corollary 3.5. Let $F: U^{2} \rightarrow V$ be a mapping and there exits real numbers $\lambda$ and $s$ such that

$$
\| F(x, y, z, w)) \|_{\alpha}^{+} \preceq \begin{cases}\lambda \otimes\left\{\|x\|^{s} \oplus\|y\|^{s} \oplus\|z\|^{s} \oplus\|w\|^{s}\right\}, & s<3 \text { or } s>3 ; \\ \lambda \otimes\|x\|^{s} \otimes\|y\|^{s} \otimes\|z\|^{s} \otimes\|w\|^{s}, & s<\frac{3}{4} \text { or } s>\frac{3}{4} \\ \lambda \otimes\left\{\|x\|^{s} \otimes\|y\|^{s} \otimes\|z\|^{s} \otimes\|w\|^{s}\right. & \\ \left.\oplus\left\{\|x\|^{4 s} \oplus\|y\|^{4 s} \oplus\|w\|^{4 s} \oplus\|z\|^{4 s}\right\}\right\}, & s<\frac{3}{4} \text { or } s>\frac{3}{4}\end{cases}
$$

for all $x, y, z, w \in U$, then there exists a unique 2- variable cubic function $C: U^{2} \rightarrow V$ such that

$$
\|f(2 x, 2 x)-2 f(x, x)-C(x, x)\|_{\alpha}^{+} \preceq\left\{\begin{array}{l}
\lambda_{\alpha}^{+} \otimes \frac{2^{(s-1)}\left(18 \oplus 2^{s+1}\right)\left(\|x\|^{s}\right)_{\alpha}^{+}}{\left|8-2^{s}\right|}, \\
\lambda_{\alpha}^{+} \otimes \frac{2^{(4 s-1)}\left(4 \oplus 2^{2 s}\right)\left(\|\left. x\right|^{4 s}\right)_{\alpha}^{+}}{\left|8-2^{4 s}\right|} \\
\lambda_{\alpha}^{+} \otimes \frac{2^{(4 s-1)}\left(22 \oplus 2^{2 s}+2 \odot 2^{4 s}\right)\left(\|x\|^{s}\right)_{\alpha}^{+}}{\left|8-2^{4 s}\right|}
\end{array}\right.
$$

for all $x \in U$.

Proof. The proof of the corollary is similar tracing as that of Corollary 3.3.

Now, we are ready to prove the main fixed point stability results.

Theorem 3.6. Let $F: U^{2} \rightarrow V$ be a mapping for which there exist a function $\varphi: U^{4} \rightarrow F^{*}(R)$ with the conditions (3.1) and (3.17) where $\mu_{i}=2$ if $i=0$ and $\mu_{1}=\frac{1}{2}$ if $i=1$ such that the functional inequality

$$
\|F(x, y, z, w)\|_{\alpha}^{+} \preceq \varphi(x, y, z, w)_{\alpha}^{+}
$$

for all $x, y, z, w \in U$. If there exists $L=L(i)<1$ such that the function

$$
x \rightarrow \gamma(x)_{\alpha}^{+}=\frac{1}{2} \odot \psi(x)_{\alpha}^{+},
$$

has the properties (3.3) and (3.19) Then there exists a unique 2-variable additive mapping A: $U^{2} \rightarrow V$ and a unique 2-variable cubic mapping $C: U^{2} \rightarrow V$ satisfying the functional equation (1.9) and

$$
\|f(x, x)-A(x, x)-C(x, x)\|_{\alpha}^{+} \preceq \frac{1}{3} \odot \frac{L^{1-i}}{1-L} \gamma(x)_{\alpha}^{+}
$$

for all $x \in U$. The mapping $\psi(x)_{\alpha}^{+}$is defined in (3.9) for all $x \in U$.

Proof. By Theorems 3.2 and 3.4, there exists a unique 2-variable additive function $A_{1}: U^{2} \rightarrow V$ and a unique 2 -variable cubic function $C_{1}: U^{2} \rightarrow V$ such that

$$
\left\|f(2 x, 2 x)-8 f(x, x)-A_{1}(x, x)\right\|_{\alpha}^{+} \preceq \frac{L^{1-i}}{1-L} \gamma(x)_{\alpha}^{+}
$$

and

$$
\left\|f(2 x, 2 x)-2 f(x, x)-C_{1}(x, x)\right\|_{\alpha}^{+} \preceq \frac{L^{1-i}}{1-L} \gamma(x)_{\alpha}^{+}
$$


for all $x \in U$. Now from (3.28) and (3.29), one can see that

$$
\begin{aligned}
\| f & (x, x)+\frac{1}{6} A_{1}(x, x)-\frac{1}{6} C_{1}(x, x) \|_{\alpha}^{+} \\
& =\left\|\left\{-\frac{f(2 x, 2 x)}{6}+\frac{8 f(x, x)}{6}+\frac{A_{1}(x, x)}{6}\right\}+\left\{\frac{f(2 x, 2 x)}{6}-\frac{2 f(x, x)}{6}-\frac{C_{1}(x, x)}{6}\right\}\right\|_{\alpha}^{+} \\
& \leq \frac{1}{6} \odot\left\{\left\|f(2 x, 2 x)-8 f(x, x)-A_{1}(x, x)\right\|_{\alpha}^{+}+\left\|f(2 x, 2 x)-2 f(x, x)-C_{1}(x, x)\right\|_{\alpha}^{+}\right\} \\
& \leq \frac{1}{6} \odot\left\{\frac{L^{1-i}}{1-L} \gamma(x)_{\alpha}^{+}+\frac{L^{1-i}}{1-L} \gamma(x)_{\alpha}^{+}\right\}
\end{aligned}
$$

for all $x \in U$. Thus we obtain (3.27) by defining $A(x, x)=\frac{-1}{6} A_{1}(x, x)$ and $C(x, x)=\frac{1}{6} C_{1}(x, x)$ ,$\psi(x)_{\alpha}^{+}$is defined in (3.9) for all $x \in U$.

The following Corollary is an immediate consequence of Theorem 3.6, using Corollaries 3.3 and 3.5 concerning the stability of (1.9).

Corollary 3.7. Let $F: U^{2} \rightarrow V$ be a mapping and there exits real numbers $\lambda$ and $s$ such that

$$
\| F(x, y, z, w)) \|_{\alpha}^{+} \preceq \begin{cases}\lambda \otimes\left\{\|x\|^{s} \oplus\|y\|^{s} \oplus\|z\|^{s} \oplus\|w\|^{s}\right\}, & s \neq 1,3 ; \\ \lambda \otimes\|x\|^{s} \otimes\|y\|^{s} \otimes\|z\|^{s} \otimes\|w\|^{s}, & s \neq \frac{1}{4}, \frac{3}{4} ; \\ \lambda \otimes\left\{\|x\|^{s} \otimes\|y\|^{s} \otimes\|z\|^{s} \otimes\|w\|^{s}\right. & \\ \left.\oplus\left\{\|x\|^{4 s} \oplus\|y\|^{4 s} \oplus\|w\|^{4 s} \oplus\|z\|^{4 s}\right\}\right\}, & s \neq \frac{1}{4}, \frac{3}{4} ;\end{cases}
$$

for all $x, y, z, w \in U$, then there exists a unique 2-variable additive mapping $A: U^{2} \rightarrow V$ and a unique 2-variable cubic mapping $C: U^{2} \rightarrow V$ such that

$$
\begin{aligned}
& \|f(x, x)-A(x, x)-C(x, x)\| \\
& \leq\left\{\begin{array}{l}
\lambda_{\alpha}^{+} \otimes \frac{2^{(s-1)}\left(18 \oplus 2^{s+1}\right)}{3}\left(\frac{1}{\left|2-2^{s}\right|}+\frac{1}{\left|8-2^{s}\right|}\right)\left(\|x\|^{s}\right)_{\alpha}^{+}, \\
\lambda_{\alpha}^{+} \otimes \frac{2^{(4 s-1)}\left(4 \oplus 2^{2 s}\right)}{3}\left(\frac{1}{\left|2-2^{s}\right|}+\frac{1}{\left|8-2^{s}\right|}\right)\left(\|x\|^{4 s}\right)_{\alpha}^{+}, \\
\lambda_{\alpha}^{+} \otimes \frac{2^{(4 s-1)}\left(22 \oplus 2^{2 s} \oplus 2 \odot 2^{4 s}\right)}{3}\left(\frac{1}{\left|2-2^{s}\right|}+\frac{1}{\left|8-2^{s}\right|}\right)\left(\|x\|^{4 s}\right)_{\alpha}^{+}
\end{array}\right.
\end{aligned}
$$

for all $x \in U$.

\section{REFERENCES}

[1] J. Aczel and J. Dhombres, Functional Equations in Several Variables, Cambridge Univ, Press, 1989.

[2] T. Aoki, On the stability of the linear transformation in Banach spaces, J. Math. Soc. Japan, 2 (1950), 64-66.

[3] M. Arunkumar, Matina J. Rassias, Yanhui Zhang, Ulam - Hyers stability of a 2- variable AC - mixed type functional equation: direct and fixed point methods, Journal of Modern Mathematics Frontier (JMMF), 2012, Vol 1 (3), 10-26.

[4] M. Arunkumar, S. Hema latha, C. Devi Shaymala Mary, Functional equation originating from arithmetic Mean of consecutive terms of an arithmetic Progression are stable in banach space: Direct and fixed point method, JP Journal of Mathematical Sciences Vol 3(1), 2012, 27-43.

[5] H.Y. Chu and D.S. Kang, On the stability of an $n-$ dimensional cubic functional equation, J. Math. Anal. Appl., 325 No. 1 (2007), 595-607.

[6] J.H. Bae and W.G. Park , A functional equation orginating from quadratic forms, J. Math. Anal. Appl. 326 (2007), 1142-1148. 
[7] S. Czerwik, Functional Equations and Inequalities in Several Variables, World Scientific, River Edge, NJ, 2002.

[8] C. Felbin, Finite dimensional fuzzy normed linear spaces, Fuzzy Sets Syst 48 (1992), 239-248.

[9] P. Gavruta, A generalization of the Hyers-Ulam-Rassias stability of approximately additive mappings , J. Math. Anal. Appl., 184 (1994), 431-436.

[10] M. Eshaghi Gordji, H. Khodaie, Solution and stability of generalized mixed type cubic, quadratic and additive functional equation in quasi-Banach spaces, arxiv: 0812. 2939v1 Math FA, 15 Dec 2008.

[11] M. Eshaghi Gordji, H. Khodaei, J.M. Rassias, Fixed point methods for the stability of general quadratic functional equation, Fixed Point Theory 12 (2011), no. 1, 71-82.

[12] T. Gantner, R. Steinlage, R. Warren, Compactness in fuzzy topological spaces, J. Math. Anal. Appl. 62 (1978) 547-562.

[13] D.H. Hyers, On the stability of the linear functional equation, Proc.Nat. Acad.Sci.,U.S.A.,27 (1941) 222224.

[14] U. Hoehle, Fuzzy real numbers as Dedekind cuts with respect to a multiple-valued logic, Fuzzy Sets Syst. 24 (1987) 263-278.

[15] D.H. Hyers, G. Isac, Th.M. Rassias, Stability of functional equations in several variables,Birkhauser, Basel, 1998.

[16] S.M. Jung, Hyers-Ulam-Rassias Stability of Functional Equations in Mathematical Analysis, Hadronic Press, Palm Harbor, 2001.

[17] Y.S.Jung and I.S.Chang, The stability of a cubic type functional equation with the fixed point alternative , J. Math. Anal. Appl. 306 (2005), 752-760.

[18] Pl. Kannappan, Functional Equations and Inequalities with Applications, Springer Monographs in Mathematics, 2009.

[19] O. Kaleva and S. Seikkala, On fuzzy metric spaces, Fuzzy Sets Syst. 12 (1984), 215-229.

[20] O. Kaleva, The completion of fuzzy metric spaces, J. Math. Anal. Appl. 109 (1985), 194-198.

[21] O. Kaleva, A comment on the completion of fuzzy metric spaces, Fuzzy Sets Syst. 159(16) (2008), 21902192.

[22] H.A. Kenary, S.Y. Jang, C. Park Fixed point approach to the Hyers-Ulam stability of a functional equation in various normed spaces, Fixed Point Theory and Applications, doi:10.1186/1687-1812-2011-67.

[23] R. Lowen, Fuzzy Set Theory, (Ch. 5 : Fuzzy real numbers), Kluwer, Dordrecht, 1996.

[24] B.Margoils, J.B.Diaz, A fixed point theorem of the alternative for contractions on a generalized complete metric space, Bull.Amer. Math. Soc. 12674 (1968), 305-309.

[25] F. Moradlou, S. Rezaee, I. Sadeqi, stability of Cauchy functional equation in Felbin's type spaces: A fixed point approach Iranian Journal of Fuzzy Systems, (Submitted).

[26] A.K. Mirmostafaee, M.S. Moslehian Fuzzy version of Hyers-Ulam-Rassias theorem, Fuzzy sets Syst. 159(6) 2008, 720-729.

[27] V.Radu, The fixed point alternative and the stability of functional equations, in: Seminar on Fixed Point Theory Cluj- Napoca, Vol. IV, 2003, in press.

[28] J.M. Rassias, On approximately of approximately linear mappings by linear mappings, J. Funct. Anal. USA, 46, (1982) 126-130.

[29] J.M. Rassias, K.Ravi, M.Arunkumar and B.V.Senthil Kumar, Ulam Stability of Mixed type Cubic and Additive functional equation, Functional Ulam Notions (F.U.N) Nova Science Publishers, 2010, Chapter $13,149-175$.

[30] J.M. Rassias, E. Son, H.M. Kim, On the Hyers-Ulam stability of $3 D$ and $4 D$ mixed type mappings, Far East J. Math. Sci. 48 (2011), no. 1, 83-102.

[31] M.M. Pourpasha, J. M. Rassias, R. Saadati, S.M. Vaezpour, A fixed point approach to the stability of Pexider quadratic functional equation with involution J. Inequal. Appl. 2010, Art. ID 839639, 18 pp.

[32] Th.M. Rassias, On the stability of the linear mapping in Banach spaces, Proc.Amer.Math. Soc., 72 (1978), 297-300.

[33] Th.M. Rassias, Functional Equations, Inequalities and Applications, Kluwer Acedamic Publishers, Dordrecht, Bostan London, 2003.

[34] K. Ravi, M. Arunkumar, On a n-dimensional additive Functional Equation with fixed point Alternative, Proceedings of International Conference on Mathematical Sciences 2007, Malaysia.

[35] K.Ravi and M.Arunkumar, Stability of a 3-variable Quadratic Functional Equation, Journal of Quality Measurement and Analysis, July 4 (1), 2008, 97-107. 
[36] K. Ravi, M. Arunkumar and J.M. Rassias, On the Ulam stability for the orthogonally general EulerLagrange type functional equation, International Journal of Mathematical Sciences, Autumn 2008 Vol.3, No. 08, 36-47.

[37] K. Ravi, J.M. Rassias, M. Arunkumar, R. Kodandan, Stability of a generalized mixed type additive, quadratic, cubic and quartic functional equation, J. Inequal. Pure Appl. Math. 10 (2009), no. 4, Article 114, $29 \mathrm{pp}$.

[38] J.M. Rassias, R. Saadati, G. Sadeghi, J. Vahidi, On nonlinear stability in various random normed spaces, Journal of Inequalities and Applications, doi:10.1186/1029-242X-2011-62

[39] S.E. Rodabaugh, Fuzzy addition in the L-fuzzy real line, Fuzzy Sets Syst. 8 (1982) 39-51.

[40] S.M. Jung, J.M. Rassias, A fixed point approach to the stability of a functional equation of the spiral of Theodorus, Fixed Point Theory Appl. 2008, Art. ID 945010, 7 pp.

[41] I. Sadeqi, F. Moradlou and M. Salehi, on approximate Cauchy equation in Felbin's type fuzzy normed linear spaces, submmitted to journal.

[42] I. Sadeqi, M. Salehi, Fuzzy compacts operators and topological degree theory, Fuzzy Sets Syst. 160(9) (2009), 1277-1285.

[43] S.M. Ulam, Problems in Modern Mathematics, Science Editions, Wiley, New York, 1964.

[44] J. Xiao, X. Zhu, On linearly topological structure and property of fuzzy normed linear space, Fuzzy Sets Syst. 125 (2002), 153-161.

[45] J. Xiao, X. Zhu, Topological degree theory and fixed point theorems in fuzzy normed space, Fuzzy Sets Syst. 147 (2004), 437-452.

[46] T.Z. Xu, J.M. Rassias, W.X Xu, Generalized Ulam-Hyers stability of a general mixed AQCQ-functional equation in multi-Banach spaces: a fixed point approach, Eur. J. Pure Appl. Math. 3 (2010), no. 6, 1032-1047.

[47] T.Z. Xu, J.M. Rassias, M.J. Rassias, W.X. Xu, A fixed point approach to the stability of quintic and sextic functional equations in quasi- $\beta$-normed spaces, J. Inequal. Appl. 2010, Art. ID 423231, 23 pp.

[48] T.Z. Xu, J.M Rassias, W.X. Xu, A fixed point approach to the stability of a general mixed AQCQfunctional equation in non-Archimedean normed spaces, Discrete Dyn. Nat. Soc. 2010, Art. ID 812545, 24 pp.

Received: May 24, 2013 